\title{
Predictors of Response to Radioligand Therapy of Metastatic Castrate-Resistant Prostate Cancer with ${ }^{177}$ Lu-PSMA-617
}

\author{
Justin Ferdinandus ${ }^{1}$, Elisabeth Eppard ${ }^{1}$, Florian C. Gaertner ${ }^{1}$, Stefan Kürpig ${ }^{1}$, Rolf Fimmers ${ }^{2}$, Anna Yordanova ${ }^{1}$, \\ Stefan Hauser ${ }^{3}$, Georg Feldmann ${ }^{4}$, Markus Essler ${ }^{1}$, and Hojjat Ahmadzadehfar ${ }^{1}$ \\ ${ }^{I}$ Department of Nuclear Medicine, University Hospital Bonn, Bonn, Germany; ${ }^{2}$ Institute for Medical Biometry, Informatics and \\ Epidemiology, University of Bonn, Bonn, Germany; ${ }^{3}$ Department of Urology, University Hospital Bonn, Bonn, Germany; and \\ ${ }^{4}$ Department of Internal Medicine, MED III, University Hospital Bonn, Bonn, Germany
}

\begin{abstract}
Radioligand therapy (RLT) with ${ }^{177}$ Lu-PSMA-617 (PSMA is prostatespecific membrane antigen) is a novel targeted therapy for metastatic prostate cancer. In this study, we evaluated the effect of different pretherapeutic parameters on the therapeutic response measured by prostate-specific antigen (PSA) 2 mo after RLT. Methods: RLT was performed in 40 hormone-refractory patients with distant metastases and progressive disease (mean age, $71.4 \mathrm{y}$ ). ${ }^{68} \mathrm{Ga}-\mathrm{PSMA}-11 \mathrm{PET} / \mathrm{CT}$ was performed in all patients $1-2$ wk before $\mathrm{RLT}$. All patients were treated with a mean of $6 \mathrm{GBq}$. The SUV $\mathrm{V}_{\max }$ of tumor lesions was determined using region-of-interest analysis. Complete blood counts, renal and liver function assessments, previous therapies, pain medication, and SUVs were included in the analysis. PSA was assessed 2 mo after RLT. Results: In the univariate analysis, younger age, higher levels of $\gamma$-glutamyl transferase, lower pretherapeutic hemoglobin, a higher Gleason score, a higher number of platelets, higher $\mathrm{C}$-reactive protein, regular need for pain medication, and higher lactate dehydrogenase had a negative impact on the therapeutic response; however, the multivariate analysis revealed that the most significant independent factors were the number of platelets and regular need for pain medication. The response was independent of the amount of PSMA uptake as well as previous therapies and other measured factors. Conclusion: A PSA decline of more than $50 \%$ was observed significantly more in patients without a regular need for analgesics.
\end{abstract}

Key Words: PSMA; ${ }^{177} \mathrm{Lu}$; prostate cancer; radioligand therapy; PSA; treatment response

J Nucl Med 2017; 58:312-319

DOI: 10.2967/jnumed.116.178228

\section{$\mathbf{P}$} rostate-specific membrane antigen (PSMA) is an attractive target for the diagnosis and therapy of metastasized prostate cancer (PC) $(1,2)$. All of the recently published papers about radioligand therapy (RLT) with ${ }^{177}$ Lu-PSMA-617 have indicated that this therapy is safe and has a low toxicity profile (3-8). The first results showed a positive effect on the prolongation of overall survival as well (6). Although, at the moment, RLT is performed for hormone-refractory patients in an advanced stage of disease with continuously increasing prostate-specific antigen (PSA), a decline in

Received May 16, 2016; revision accepted Aug. 10, 2016.

For correspondence or reprints contact: Hojjat Ahmadzadehfar, University Hospital Bonn, Department of Nuclear Medicine, Sigmund-Freud-Strasse 25, 53127 Bonn, Germany.

E-mail: hojjat.ahmadzadehfar@ukb.uni-bonn.de

Published online Sep. 1, 2016.

COPYRIGHT (C) 2017 by the Society of Nuclear Medicine and Molecular Imaging.
PSA has been detected in about $60 \%-70 \%$ of patients (3-8). However, it is still unclear as to whether any of the pretherapeutic parameters, such as the level of PSMA expression in ${ }^{68} \mathrm{Ga}$-PSMA-11 PET or prior therapies, may affect the treatment response according to PSA changes. In the current study, we retrospectively evaluated the effect of different pretherapeutic parameters and prior therapies on the therapeutic response measured by PSA 2 mo after the first cycle of RLT.

\section{MATERIALS AND METHODS}

\section{Patients}

Forty consecutive hormone-refractory PC patients with distant metastases and progressive disease according to the PSA level were treated with ${ }^{177} \mathrm{Lu}-\mathrm{PSMA}-617$. The data regarding the treatment response and toxicity as well as treatment tolerability of the included patients in this study have been published together with the data from 3 other centers as a multicenter study (7). The prior therapies are listed in Table 1. All patients had an extensive metastatic disease, which is demonstrated in Table 1. PSMA PET was performed for all patients 1-2 wk before therapy to evaluate the PSMA expression status of the metastases. The local ethics committee approved this retrospective study, and all subjects signed a written informed consent form.

\section{Treatment Planning}

${ }^{68} \mathrm{Ga}$-PSMA-11 PET/CT. ${ }^{68} \mathrm{Ga}-\mathrm{PSMA}-11$ was applied via slow intravenous injection (30-60 s) using a weight-adapted dose of $2 \mathrm{MBq} / \mathrm{kg}$ of body weight in a total volume of $5-10 \mathrm{~mL}$ (diluted with $0.9 \%$ sterile sodium chloride solution), followed by $20 \mathrm{~mL}$ of sterile $0.9 \%$ sodium chloride. The average injected dose was $140 \mathrm{MBq}$. PET/CT was performed on a Biograph 2 PET/CT scanner (Siemens Medical Solutions). PET emission data were acquired from the pelvis to the head using $6-8$ bed positions (3-dimensional mode); the emission time was 4 min per bed position. PET images were reconstructed iteratively. After reconstruction, images were smoothed by a 5-mm gaussian filter. Attenuation correction was performed using CT data. The $\mathrm{SUV}_{\max }$ was determined by a region of interest applied in the transaxial attenuation-corrected PET slice with the

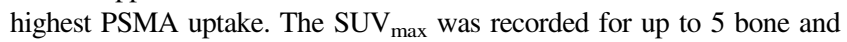
lymph node metastases as well as for liver and spleen for the calculation of target/nontarget. Thus, the following parameters were calculated: mean $\mathrm{SUV}_{\max }$ of the bone lesions $\left(\mathrm{SUV}_{\max } \mathrm{Bn}=\mathrm{SUV}_{\max } \mathrm{Bn}_{1}+\ldots . . \mathrm{SUV}_{\max } \mathrm{Bn} / \mathrm{x}\right)$, mean $\mathrm{SUV}_{\max }$ of the lymph node metastases $\left(\mathrm{SUV}_{\max } \mathrm{Ln}=\right.$ $\left.\mathrm{SUV}_{\max } \operatorname{Ln}_{1}+\ldots . . \mathrm{SUV}_{\max } \operatorname{Ln}_{\mathrm{x}} / \mathrm{x}\right), \mathrm{SUV}_{\max } \mathrm{Bn} / \mathrm{SUV}_{\max } \mathrm{Liver}_{\mathrm{m}} \mathrm{SUV}_{\max } \mathrm{Bn} /$ $\mathrm{SUV}_{\max }$ spleen, $\mathrm{SUV}_{\max } \mathrm{Ln} / \mathrm{SUV}_{\max }$ liver, $\mathrm{SUV}_{\max } \mathrm{Ln} / \mathrm{SUV}_{\max }$ spleen.

\section{Laboratory Tests}

One day before therapy, the hematologic and renal status, liver function tests, tumor marker PSA, alkaline phosphatase, and blood biochemistry were evaluated in all patients (Tables 2 and 3). 
TABLE 1

Patient Characteristics

\begin{tabular}{|c|c|c|c|c|c|}
\hline Characteristic & No. of patients & History $(n)$ & Ongoing $(n)$ & No history $(n)$ & Extent \\
\hline \multicolumn{6}{|l|}{ Gleason score } \\
\hline 6 & $4(10)$ & & & & \\
\hline 7 & $3(7.5)$ & & & & \\
\hline 8 & $11(27.5)$ & & & & \\
\hline 9 & $14(35)$ & & & & \\
\hline 10 & $5(12.5)$ & & & & \\
\hline Unknown & $3(7.5)$ & & & & \\
\hline \multicolumn{6}{|l|}{ Prior and ongoing therapies } \\
\hline Prostatectomy & & $21(52.5)$ & & $19(47.5)$ & \\
\hline Abiraterone ${ }^{* \dagger}$ & & $18(45)$ & $9(22.5)$ & $13(32.5)$ & \\
\hline Enzalutamide ${ }^{\star \dagger}$ & & $10(25)$ & $15(37.5)$ & $15(37.5)$ & \\
\hline Chemotherapy & & $21(52.5)$ & & $19(47.5)$ & \\
\hline $\begin{array}{l}\text { Bisphosphonate or RANKL } \\
\text { inhibitor }\end{array}$ & & $3(7.5)$ & $29(72.5)$ & $8(20)$ & \\
\hline${ }^{223} \mathrm{Ra}$ & & $14(35)$ & & $26(65)$ & \\
\hline Regular need for analgesics & & & $15(37.5)$ & & \\
\hline \multicolumn{6}{|c|}{$\begin{array}{l}\text { Extent of disease in } 40 \text { patients, } \\
\text { detected by }{ }^{68} \mathrm{Ga} \text {-PSMA PET/CT }\end{array}$} \\
\hline Local recurrence & $17(42.5)$ & & & & \\
\hline \multirow[t]{4}{*}{ Bone met } & $40(100)$ & & & & $<6$ met in 3 pts $(7.5)$ \\
\hline & & & & & $6-20$ met in 2 pts (5) \\
\hline & & & & & $>20$ met in 29 pts (72.5) \\
\hline & & & & & Diffuse met in 6 pts (15) \\
\hline \multirow[t]{3}{*}{ Lymph node met } & $33(82.5)$ & & & & Ileal and abd in 14 pts (45.5) \\
\hline & & & & & Thor in 2 pts (6) \\
\hline & & & & & Ileal to thor in 16 pts $(48.5)$ \\
\hline \multirow[t]{2}{*}{ Liver met } & $8(20)$ & & & & Singular met in 2 pts \\
\hline & & & & & Multiple met in 6 pts \\
\hline \multirow[t]{2}{*}{ Lung met } & $3(7.5)$ & & & & Singular met in 2 pts \\
\hline & & & & & Multiple met in $1 \mathrm{pt}$ \\
\hline \multicolumn{6}{|c|}{$\begin{array}{l}\text { *Five patients took neither abiretarone nor enzalutamide, and } 3 \text { patients took bicalutamide. } \\
{ }^{\dagger} \text { All patients who were taking enzalutamide or abiraterone had increasing PSA under these medications. } \\
\text { RANKL = receptor activator of nuclear factor K-B ligand; met = metastases; pts = patients; abd = abdominal; thor = thoracic. } \\
\text { Data in parentheses are percentages. }\end{array}$} \\
\hline
\end{tabular}

\section{RLT}

PSMA ligand (PSMA-617) was obtained from ABX GmbH. The preparation of ${ }^{177} \mathrm{Lu}-\mathrm{PSMA}-617$ was explained in detail in a previous publication (3).

The treatment solution was administered by slow intravenous injection over $1 \mathrm{~min}$ followed by $1,000 \mathrm{~mL}$ of $\mathrm{NaCl}$ or Ringer's. All patients were discharged $48 \mathrm{~h}$ after therapy according to the rules of the Federal Office for Radiation Protection in Germany (BfS).

\section{Tumor Response Evaluation}

The tumor marker PSA was used as the main marker for the response evaluation. We classified the changes in PSA level as a decrease of more than $50 \%$ and any decline. Any increase in PSA was considered as disease progression.

\section{Evaluated Parameters for Evaluation of Predictive Factors of Response}

All of the patients' parameters and laboratory tests are shown in Tables 1-4, including the extent of bone and lymph node metastases, the existence of liver metastases, age, the need for pain medication, the Eastern Cooperative Oncology Group (ECOG) score, SUV, SUV ${ }_{\max } \mathrm{Bn}$, $\mathrm{SUV}_{\max } \mathrm{Ln}, \quad \mathrm{SUV}_{\max } \mathrm{BN} /$ liver, $\quad \mathrm{SUV}_{\max } \mathrm{BN} /$ spleen, $\quad \mathrm{SUV}_{\max } \mathrm{Ln} / \mathrm{liver}$; $\mathrm{SUV}_{\max } \mathrm{Ln} / \mathrm{spleen}$, injected dose of ${ }^{177} \mathrm{Lu}-\mathrm{PSMA}-617$, and injected dose $/ \mathrm{kg}$ of body weight.

\section{Statistical Analysis}

The baseline characteristics of the study were analyzed with regard to their correlation to quantitative PSA changes. For this, a linear regression was used. Two qualitative assessments of PSA decline (any PSA decline and PSA decline $>50 \%$ ) were analyzed with uni- and 
TABLE 2

Blood, Renal, and Hepatic Parameters Before First Cycle

\begin{tabular}{|c|c|c|c|c|}
\hline Parameter & Minimum & Maximum & Mean & Median \\
\hline \multicolumn{5}{|l|}{ Blood parameters } \\
\hline WBCs (G/L) (norm, 3.6-10.5) & 2.7 & 12.2 & 6.4 & 6.3 \\
\hline $\mathrm{Hb}(\mathrm{g} / \mathrm{dL})($ norm, $12.5-17.2)$ & $6.0^{*}$ & 14.5 & 10.9 & 11.0 \\
\hline Plt (G/L) (norm, 160-370) & 62 & 557 & 278.9 & 262.5 \\
\hline \multicolumn{5}{|l|}{ Renal parameters } \\
\hline Creatinine $(\mathrm{mg} / \mathrm{dL})$ (norm, 0.6-1.3) & 0.53 & 2.54 & 0.95 & 0.9 \\
\hline GFR (mL/min) (norm, $>70)$ & 50 & $>70^{\dagger}$ & & \\
\hline \multicolumn{5}{|l|}{ Liver function tests } \\
\hline Bilirubin total (mg/dL) (norm, 0.2-1.0) & 0.21 & 1.20 & 0.41 & 0.40 \\
\hline ALT (U/L) (norm, <50) & 8 & 67 & 18.9 & 16.5 \\
\hline AST(U/L) (norm, <50) & 10 & 302 & 37.5 & 24 \\
\hline GGT (U/L) (norm, <55) & 17 & 425 & 67.6 & 40 \\
\hline Albumin (g/L) (norm, 35-52) & 25.8 & 46.9 & 37.6 & 37.6 \\
\hline
\end{tabular}

*Seven patients had received blood transfusion before therapy because of grade 3 tumor anemia $10-180 \mathrm{~d}$ before ${ }^{177}$ Lu-PSMA therapy (median, $21 \mathrm{~d}$ ); 1 of the patients had another blood transfusion $1 \mathrm{~d}$ before therapy because of an $\mathrm{Hb}$ of $6 \mathrm{~g} / \mathrm{dL}$.

${ }^{\dagger}$ Thirty-two patients (80\%) had a GFR greater than $70 \mathrm{~mL} / \mathrm{min}$.

WBCs = white blood cells; $\mathrm{Hb}=$ hemoglobin; Plt = platelets; GFR = glomerular fraction rate; $\mathrm{ALT}=$ alanine transaminase; $\mathrm{AST}=$ aspartate transaminase; GGT = y-glutamyl transferase.

multivariate logistic regression. Multivariate models were derived by stepwise regression analysis primarily including parameters selected depending on the results of the unifactoral logistic regression.

\section{RESULTS}

Forty cycles of ${ }^{177} \mathrm{Lu}-\mathrm{PSMA}-617$ were performed in 40 consecutive hormone-refractory patients with a mean age of $71.4 \mathrm{y}$ (age range, 43-87 y). Each patient received a mean of $6.0 \mathrm{GBq}$ (range, 4.1-7.1 GBq; median, 6.1 GBq) of ${ }^{177} \mathrm{Lu}-\mathrm{PSMA}-617$. According to the patient's weight, each patient received a mean activity of $78.5 \mathrm{MBq} / \mathrm{kg}$ of body weight. The mean and median time between diagnosis of PC and RLT was 6.9 and 5.5 y (range, 0.8-20 y), respectively. The median Gleason score was 9 (range, 6-10). The extent of disease in these patients and their prior and ongoing therapies are shown in Table 1. The mean and median PSA levels before therapy were 495.1 and $325.5 \mathrm{ng} / \mathrm{mL}$ (range, 4.73-2,360 $\mathrm{ng} / \mathrm{mL}$ ), respectively (Table 3 ).

TABLE 3

Tumor Parameters

\begin{tabular}{ccccc}
\hline Parameter & Minimum & Maximum & Mean & Median \\
\hline $\begin{array}{c}\text { ALP (U/L) } \\
(\text { norm, 34-117) }\end{array}$ & 38 & 1,607 & 247.3 & 176.5 \\
$\begin{array}{c}\text { LDH (U/L) } \\
(\text { norm, }<248)\end{array}$ & 147 & 1,875 & 411.4 & 257 \\
$\begin{array}{c}\text { CRP }(\mathrm{mg} / \mathrm{L}) \\
(\mathrm{norm},<3)\end{array}$ & 0.2 & 128 & 24.9 & 16.2 \\
$\quad \mathrm{PSA}(\mathrm{ng} / \mathrm{mL})$ & 4.73 & 2,360 & 495.1 & 325.5 \\
\hline & & & & \\
\hline ALP = alkaline phosphatase; LDH = lactate dehydrogenase. \\
\hline
\end{tabular}

Twenty-six patients exhibited good ECOG scores ( 0 or 1$)$. Thirteen patients exhibited an ECOG score of 2, and 1 an ECOG score of 3 . The blood and renal parameters before the first cycle are shown in Table 2. Seven patients had received blood transfusion before therapy because of grade 3 tumor anemia 10-180 d before ${ }^{177} \mathrm{Lu}-\mathrm{PSMA}-617$ therapy (median, $21 \mathrm{~d}$ ). The tumor parameters are listed in Table 3. The SUVs of bone and lymph node metastases are shown in Table 4. Eight weeks after RLT, 27 of 40 patients $(67.5 \%)$ experienced a PSA decline, of whom 13 showed a PSA decline of more than $50 \%$ (32.5\%). Thirteen patients showed progressive disease according to an increasing PSA level (Supplemental Fig. 1; supplemental materials are available at http://jnm.snmjournals.org).

\section{Impact of Pretherapeutic Parameters on Percentage PSA Changes}

The linear regression analysis of different parameters showed that a higher platelets count, high C-reactive protein (CRP) level, younger age, higher Gleason score, and a regular need for pain medication have a negative impact on the PSA response according

TABLE 4

SUVs of 40 Patients Before ${ }^{177}$ Lu-PSMA Therapy

\begin{tabular}{lcrrr}
\hline \multicolumn{1}{c}{ Parameter } & Minimum & Maximum & Mean & Median \\
\hline SUVmaxBN & 4.50 & 100.68 & 26.07 & 20.76 \\
\hline SUVmaxBN/liver & 0.60 & 13.51 & 4.17 & 3.66 \\
\hline SUVmaxBN/spleen & 0.69 & 19.55 & 4.27 & 2.98 \\
SUVmaxLn & 4.0 & 98.60 & 26.24 & 14.10 \\
\hline SUVmaxLn/liver & 0.41 & 15.91 & 3.76 & 2.53 \\
SUVmaxLn/spleen & 0.38 & 33.65 & 4.95 & 2.10 \\
\hline
\end{tabular}


TABLE 5

Linear Regression Analysis of Different Pretherapeutic Parameters and Their Impact on PSA Change

\begin{tabular}{|c|c|c|c|}
\hline Parameter & $P$ & Parameter & $P$ \\
\hline Amount of activity (MBq) & NS & Creatinine & NS \\
\hline Activity (MBq)/weight (Kg) & NS & Total bilirubin & NS \\
\hline Abiretarone & NS & $\mathrm{Hb}$ & NS \\
\hline Enzalutamide & NS & $\mathrm{RBC}$ & NS \\
\hline Chemotherapy & NS & WBCs & NS \\
\hline${ }^{223} \mathrm{Ra}$ & NS & PSA level & NS \\
\hline Bisphosphonate or denosumab & NS & SUV $_{\max }($ bone) & NS \\
\hline Number of bone metastases & NS & SUV $_{\max }$ (lymph node) & NS \\
\hline Extent of lymph node metastases & NS & SUV $_{\max }$ bone/liver & NS \\
\hline Existence of liver metastases & NS & $\mathrm{SUV}_{\max }$ bone/spleen & NS \\
\hline ECOG performance status & NS & SUV $_{\max }$ lymph node/liver & NS \\
\hline Albumin & NS & SUV $_{\max }$ lymph node/spleen & NS \\
\hline AST and ALT & NS & Plt & 0.0005 \\
\hline ALP & NS & CRP & 0.0203 \\
\hline GGT & NS & Age & 0.0139 \\
\hline LDH & NS & Gleason score & 0.0096 \\
\hline GFR & NS & Regular need for pain medication & 0.0050 \\
\hline
\end{tabular}

$\mathrm{NS}=$ not significant; $\mathrm{Hb}=$ hemoglobin; RBC = red blood cells; WBCs = white blood cells; AST = aspartate aminotransferase; ALT = alanine transaminase; PIt = platelets; ALP = alkaline phosphatase; $\mathrm{LDH}=$ lactate dehydrogenase; GFR = glomerular filtration rate.

to percentage PSA changes (Table 5). Figure 1 shows the significant correlation between age and better PSA response $\left(r^{2}=0.149\right.$; $P=0.013)$, and Figure 2 shows the significant correlation between the platelet count and worse PSA response $\left(r^{2}=0.274 ; P=0.0005\right)$.

\section{Impact of Pretherapeutic Parameters on Any PSA Decline}

The univariate analysis showed that higher levels of $\gamma$-glutamyl transferase (GGT) $(>100 \mathrm{U} / \mathrm{L} ; P=0.001)$, platelet counts $(>300 \mathrm{G} / \mathrm{L}$; $P=0.001)$, high CRP $(>20 \mathrm{mg} / \mathrm{L} ; P=0.006)$, a Gleason score of 10, younger age (cutoff, $65 \mathrm{y} ; P=0.001$ ), high lactate dehydrogenase, lower pretherapeutic hemoglobin, and regular need for pain medication had a negative impact on any PSA decline. The other factors, including SUVs, did not have any significant

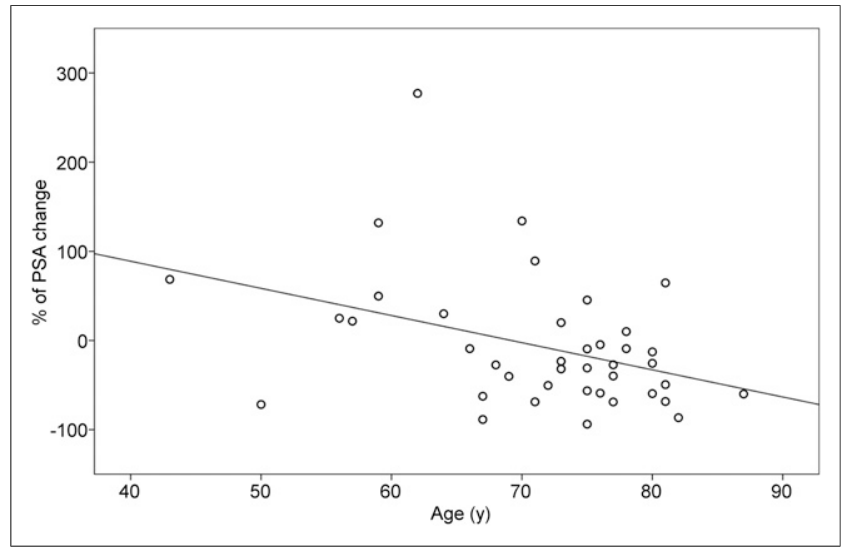

FIGURE 1. Correlation between age and percentage PSA changes $\left(R^{2}=0.149\right)$. impact on PSA (Table 6). In the multivariate analysis, only a high platelet count and regular need for pain medication kept their significance (Table 5).

\section{Impact of Pretherapeutic Parameters on PSA Decline of More Than $\mathbf{5 0 \%}$}

The univariate analysis showed that only a Gleason score of 10 and regular need for pain medication had a negative impact on PSA decline of more than $50 \%$. No patient with a Gleason score of 10 showed a PSA decline, and only 2 from 15 patients (13.3\%), who had a regular need for analgesics, showed a PSA decline of more than $50 \%$. Again, the regular need for pain medication kept its significance in the multivariate analysis (Table 7).

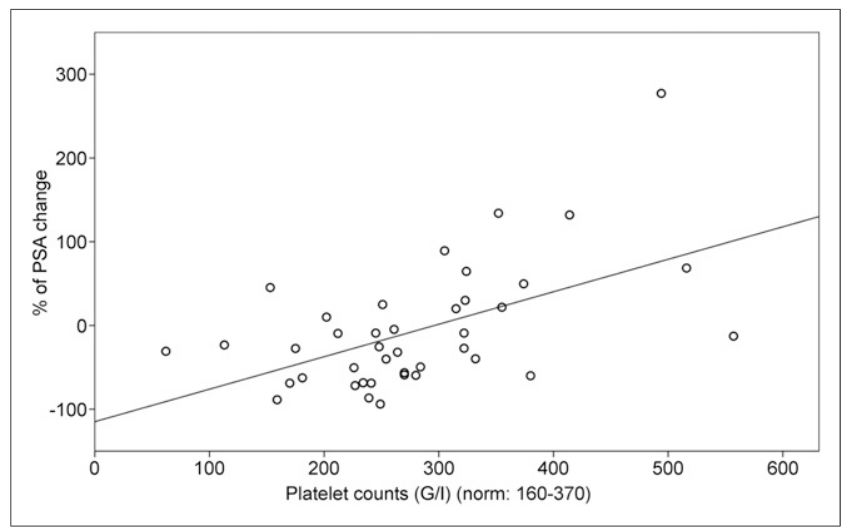

FIGURE 2. Correlation between platelet counts and percentage PSA changes $\left(R^{2}=0.274\right)$. 
TABLE 6

Univariate and Multivariate Analyses of Different Pretherapeutic Parameters Regarding Any PSA Decline

\begin{tabular}{|c|c|c|c|c|}
\hline Parameter & Odds ratio & $95 \% \mathrm{Cl}$ & $P$ & $P$ multivariate analysis \\
\hline Activity (MBq)/weight (kg) & 0.989 & $0.944-1.035$ & 0.62 & \\
\hline Abiretarone & 0.846 & $0.368-1.944$ & 0.69 & \\
\hline Enzalutamide & 0.932 & $0.398-2.181$ & 0.87 & \\
\hline Chemotherapy & 0.828 & $0.426-1.609$ & 0.57 & \\
\hline${ }^{223} \mathrm{Ra}$ & 0.436 & $0.097-1.958$ & 0.27 & \\
\hline Bisphosphonate or denosumab & 0.848 & $0.231-3.109$ & 0.80 & \\
\hline No. of bone metastases & 1.165 & $0.444-3.061$ & 0.75 & \\
\hline Extent of lymph node metastases & 1.281 & $0.731-2.243$ & 0.38 & \\
\hline Existence of liver metastases & 1.114 & $0.202-6.158$ & 0.90 & \\
\hline ECOG performance status & 1.685 & $0.761-3.733$ & 0.19 & \\
\hline Albumin & 0.987 & $0.847-1.149$ & 0.86 & \\
\hline AST & 1.018 & $0.988-1.048$ & 0.23 & \\
\hline ALT & 1.043 & $0.976-1.115$ & 0.21 & \\
\hline GGT & 1.024 & $1.006-1.041$ & 0.008 & NS \\
\hline ALP & 1.003 & $0.999-1.006$ & 0.15 & \\
\hline LDH & 1.002 & $1.000-1.005$ & 0.04 & NS \\
\hline Creatinine & 4.714 & $0.464-47.907$ & 0.19 & \\
\hline Total bilirubin & 0.014 & $<0.001-7.401$ & 0.18 & \\
\hline $\mathrm{Hb}$ & 0.574 & $0.341-0.967$ & 0.04 & NS \\
\hline $\mathrm{RBC}$ & 0.429 & $0.127-1.444$ & 0.17 & \\
\hline WBCs & 1.220 & $0.890-1.674$ & 0.21 & \\
\hline PSA level & 0.999 & $0.997-1.000$ & 0.15 & \\
\hline SUV $_{\max }($ bone) & 0.974 & $0.931-1.020$ & 0.26 & \\
\hline SUV $_{\max }$ (lymph node) & 0.980 & $0.944-1.018$ & 0.30 & \\
\hline SUV $_{\max }$ bone/liver & 0.746 & $0.529-1.053$ & 0.09 & \\
\hline $\mathrm{SUV}_{\max }$ bone/spleen & 0.635 & $0.394-1.022$ & 0.06 & \\
\hline $\mathrm{SUV}_{\max }$ lymph node/liver & 0.752 & $0.501-1.130$ & 0.16 & \\
\hline $\mathrm{SUV}_{\max }$ lymph node/spleen & 0.828 & $0.612-1.120$ & 0.22 & \\
\hline Plt & 1.010 & $1.001-1.018$ & 0.02 & 0.01 \\
\hline CRP & 1.047 & $1.011-1.085$ & 0.009 & NS \\
\hline Age & 0.885 & $0.805-0.972$ & 0.01 & NS \\
\hline Gleason score & 4.121 & $1.304-13.016$ & 0.01 & NS \\
\hline Regular need for pain medication & 0.091 & $0.017-0.501$ & 0.005 & 0.0018 \\
\hline
\end{tabular}

$\mathrm{Cl}=$ confidence interval; NS = not significant; AST = aspartate aminotransferase; ALT = alanine transaminase; ALP = alkaline phosphatase; $\mathrm{LDH}=$ lactate dehydrogenase; $\mathrm{Hb}=$ hemoglobin; $\mathrm{RBC}=$ red blood cells; $\mathrm{WBC} s=$ white blood cells; Plt $=$ platelets.

\section{DISCUSSION}

In addition to docetaxel, which until 2010 was the only drug with a positive effect on overall survival, in recent years, 5 new drugs (abiraterone, enzalutamide, sipuleucel-T, cabazitaxel, and ${ }^{223} \mathrm{Ra}$ ) have shown efficacy in prolonging survival in castration-resistant PC patients (9-14). However, despite all of these new and effective therapies, patients with hormone-refractory metastatic PC still reach an endpoint at which no approved therapy is effective or indicated.

According to retrospective studies, RLT with ${ }^{177}$ Lu-PSMA-617 has been shown to be effective, inducing PSA decline in almost $70 \%$ of patients who were in a salvage situation, with a favorable toxicity profile and mild side effects (3-8).
In our recently published study (8), we demonstrated the treatment response and side effects of ${ }^{177}$ Lu-PSMA-617 therapy in 24 patients, who were treated with up to 2 cycles. In this small group of patients, we found that the only pretherapeutic factor that had a negative impact on PSA decline was the regular use of opioids. No other pretherapeutic factors, inclusive of prior therapies, had any impact on the treatment response according to PSA decline.

We also showed the reliability of PSA for treatment follow-up. There was a significant correlation between the treatment response according to PSMA PET/CT imaging and PSA changes, which shows the value of PSA changes for the follow-up of patients on PSMA therapy. In the current study, we evaluated more parameters 
TABLE 7

Univariate and Multivariate Analysis of Different Pretherapeutic Parameters Regarding PSA Decline of More Than 50\%

\begin{tabular}{|c|c|c|c|c|}
\hline Parameter & Odds ratio & $95 \% \mathrm{Cl}$ & $P$ & $P$ multivariate analysis \\
\hline Activity (MBq)/weight (kg) & 1.028 & $0.979-1.079$ & 0.26 & \\
\hline Abiretarone & 0.986 & $0.427-2.280$ & 0.97 & \\
\hline Enzalutamide & 0.738 & $0.315-1.728$ & 0.48 & \\
\hline Chemotherapy & 1.526 & $0.775-3.005$ & 0.22 & \\
\hline${ }^{223} \mathrm{Ra}$ & 1.324 & $0.322-5.439$ & 0.69 & \\
\hline Bisphosphonate or denosumab & 1.179 & $0.322-4.320$ & 0.80 & \\
\hline No. of bone metastases & 1.351 & $0.538-3.392$ & 0.52 & \\
\hline Extent of lymph node metastases & 1.189 & $0.721-1.961$ & 0.49 & \\
\hline Existence of liver metastases & 0.661 & $0.088-4.956$ & 0.68 & \\
\hline ECOG performance status & 2.150 & $0.918-5.036$ & 0.07 & \\
\hline Albumin & 1.042 & $0.894-1.215$ & 0.59 & \\
\hline AST & 1.009 & $0.983-1.035$ & 0.49 & \\
\hline ALT & 1.017 & $0.948-1.092$ & 0.63 & \\
\hline GGT & 1.013 & $0.995-1.033$ & 0.16 & \\
\hline ALP & 1.002 & $0.998-1.006$ & 0.36 & \\
\hline LDH & 1.005 & $0.999-1.010$ & 0.12 & \\
\hline Creatinine & 3.031 & $0.218-42.194$ & 0.40 & \\
\hline Total bilirubin & 0.024 & $<0.001-1.908$ & 0.09 & \\
\hline $\mathrm{Hb}$ & 0.868 & $0.560-1.345$ & 0.52 & \\
\hline $\mathrm{RBC}$ & 1.014 & $0.326-3.158$ & 0.98 & \\
\hline WBCs & 1.377 & $0.956-1.984$ & 0.08 & \\
\hline PSA level & 1.000 & $0.999-1.001$ & 0.77 & \\
\hline SUV $_{\max }($ bone) & 1.023 & $0.979-1.070$ & 0.30 & \\
\hline SUV $_{\max }$ (lymph node) & 0.988 & $0.960-1.016$ & 0.39 & \\
\hline $\mathrm{SUV}_{\max }$ bone/liver & 1.199 & $0.900-1.597$ & 0.21 & \\
\hline $\mathrm{SUV}_{\max }$ bone/spleen & 1.120 & $0.898-1.398$ & 0.31 & \\
\hline SUV $_{\max }$ lymph node/liver & 0.971 & $0.785-1.200$ & 0.78 & \\
\hline $\mathrm{SUV}_{\max }$ lymph node/spleen & 0.990 & $0.896-1.094$ & 0.84 & \\
\hline Plt & 1.007 & $0.999-1.015$ & 0.10 & \\
\hline CRP & 1.051 & $0.995-1.110$ & 0.07 & \\
\hline Age & 0.953 & $0.876-1.036$ & 0.25 & \\
\hline Gleason score & 1.598 & $0.853-2.991$ & 0.01 & NS \\
\hline Regular need for pain medication & 0.156 & $0.036-0.669$ & 0.01 & 0.01 \\
\hline
\end{tabular}

$\mathrm{Cl}=$ confidence interval; AST = aspartate aminotransferase; ALT = alanine transaminase; ALP = alkaline phosphatase; $\mathrm{LDH}=$ lactate dehydrogenase; $\mathrm{Hb}=$ hemoglobin; RBC = red blood cells; Plt = platelets; WBCs = white blood cells; NS = not significant.

in a greater number of patients after the first cycle. The data regarding the treatment response and toxicity as well as treatment tolerability of the included patients in this study have been published together with the data from 3 other centers as a multicenter study (7). In the current study, we first tried to determine whether there was a correlation between the percentage of PSA change and any of the pretherapeutic factors (Table 5). One of the first issues for the treatment planning is the influence of prior therapies on the treatment response. Although all of our patients had received different treatments before ${ }^{177} \mathrm{Lu}-\mathrm{PSMA}-617$, in this patient collective, these therapies did not have any influence on the treatment response; however, the influence of prior therapies in combination with RLT on toxicity should be evaluated. It is still unclear whether the treatment response could be better using ${ }^{177} \mathrm{Lu}-\mathrm{PSMA}-617$ as first- or second-line therapy.

In theranostics, 2 issues should be clarified using diagnostic scans before planning radionuclide therapy: first, whether there is any tracer uptake in the tumor, and second, the amount of the uptake.

Concerning the first point, in the case of thyroid cancer or neuroendocrine tumors without any tracer uptake, ${ }^{131}$ I diagnostic scanning or somatostatin receptor imaging, respectively, is generally excluded from ${ }^{131} \mathrm{I}$ or ${ }^{177} \mathrm{Lu}$-DOTATATE therapy. This also applies to ${ }^{177} \mathrm{Lu}$-PSMA-617 therapy. Moreover, only metastases that show PSMA expression can be treated with PSMA therapy. 
The second point implies that the amount of tracer uptake is also of considerable importance, because higher radioiodine uptake predicts a better treatment response. In the case of neuroendocrine tumors, there is a positive correlation between the degree of somatostatin receptor expression and the treatment response as well as prognosis $(15,16)$; however, in the current study, the $\mathrm{SUV}_{\max }$ of ${ }^{68} \mathrm{Ga}$-PSMA-11 did not significantly influence the treatment response. One explanation for this difference between somatostatin receptor imaging and PSMA imaging may be the physiopathologic mechanism of tracer uptake. There is an inverse association between somatostatin receptor type 2A (SSTR2A) with the Ki-67 index, and SSTR2A is highly expressed in G1 and G2 neuroendocrine tumors but is significantly less abundant in neuroendocrine carcinomas (G3) (17); thus, higher expression of SSTR2A is correlated with lower grade of the neuroendocrine tumor. In contrast, the amount of PSMA expression is directly correlated with metastases, androgen independence, and progression of PC (18). More aggressive tumors may express higher PSMA levels, but despite better uptake of ${ }^{177} \mathrm{Lu}$-PSMA-617, because of the rapid growth of metastases, the response rate does not correlate with uptake. Another explanation could be the different washout times of ${ }^{177} \mathrm{Lu}$-PSMA-617 in the respective metastases. These should both be confirmed in studies with more patients.

Compared with prior therapies and the SUV of PSMA-11, there were some parameters shown to have a negative impact on the treatment response. A higher platelet count, high CRP level, younger age, higher Gleason score, and a regular need for pain medication had a negative impact on the PSA response according to percentage PSA changes. In our study, 8 patients were younger than $65 \mathrm{y}$, of whom 4 had PC with a Gleason score of 10 and the others a Gleason score of 8 or greater, which could explain the worse response in these patients.

Bensalah et al. (19) reported the prognostic value of thrombocytosis in 774 patients with renal cell carcinoma. They reported that platelet count was strongly correlated with $\mathrm{T}$ stage, Fuhrman grade, tumor size, nodal invasion, and the presence of distant metastasis. Thrombocytosis had an impact on prognosis in localized and metastatic disease. In their study on patients with a platelet count of less than $450,000 / \mathrm{mm}^{3}$, the 5-y survival rate was $70 \%$, compared with $38 \%$ when the platelet count was $450,000 / \mathrm{mm}^{3}$ or greater. The negative impact of thrombocytosis on prognosis has been also reported in other cancer types, such as bladder cancer (20), gynecologic malignancies (21-23), and gastrointestinal cancers (24), as well as in early and advanced breast cancer $(25,26)$. According to different published studies, cytokines, in particular IL-6, play an important role in the development of thrombopoiesis and thrombocytosis (22-24). Circulating tumor cells may use platelets as a protective shield against attack by the immune system and as facilitators for attachment to endothelial cells at metastatic sites $(27,28)$. Antiplatelet agents and anticoagulants have potent inhibitory effects on tumor cell platelet interactions and can prevent metastases in experimental settings in various malignancies $(27,29-32)$.

In our study, there was also a significant correlation between the number of platelets and the negative percentage change in PSA (Fig. 2). In the univariate analysis of different pretherapeutic factors regarding any PSA decline, a platelet count of more than 300 $\mathrm{G} / \mathrm{L}$ also had a negative impact on the therapeutic response in addition to high GGT (>100U/L), high CRP (>20 mg/L), and a Gleason score of 10, younger age (cutoff: $65 \mathrm{y}$ ), lower pretherapeutic hemoglobin, and regular need for pain medication; however, in the multivariate analysis, only the platelet count and regular need for pain medication retained their significance.
Regarding a PSA decline of more than $50 \%$, only a Gleason score of 10 and regular need for pain medication had a negative impact on the therapeutic response in the univariate analysis; in the multivariate analysis, only the regular need for pain medication was significant. Patients with advanced disease and high tumor load often suffer from pain, which affects their quality of life. A regular need for pain medication is an indirect sign of advanced disease, which explains its negative impact on the treatment response.

\section{CONCLUSION}

According to the results of this study, a favorable response rate (PSA decline $>50 \%$ ) may be observed significantly more in patients without a regular need for analgesics. The results of the current study should be confirmed by prospective trials especially in respect to the effect of these parameters on survival.

\section{DISCLOSURE}

No potential conflict of interest relevant to this article was reported.

\section{ACKNOWLEDGMENTS}

We are grateful to the nursing staff of the treatment ward in our department. We give special thanks to our study nurse, Ulrike Kuhn-Seifer (Department of Nuclear Medicine Bonn).

\section{REFERENCES}

1. Afshar-Oromieh A, Hetzheim H, Kratochwil C, et al. The theranostic PSMA ligand PSMA-617 in the diagnosis of prostate cancer by PET/CT: biodistribution in humans, radiation dosimetry, and first evaluation of tumor lesions. J Nucl Med. 2015;56:1697-1705.

2. Afshar-Oromieh A, Malcher A, Eder M, et al. PET imaging with a $\left[{ }^{68} \mathrm{Ga}\right]$ galliumlabelled PSMA ligand for the diagnosis of prostate cancer: biodistribution in humans and first evaluation of tumour lesions. Eur J Nucl Med Mol Imaging. 2013;40:486-495.

3. Ahmadzadehfar H, Rahbar K, Kurpig S, et al. Early side effects and first results of radioligand therapy with ${ }^{177}$ Lu-DKFZ-617 PSMA of castrate-resistant metastatic prostate cancer: a two-centre study. EJNMMI Res. 2015;5:114.

4. Baum RP, Kulkarni HR, Schuchardt C, et al. ${ }^{177}$ Lu-labeled prostate-specific membrane antigen radioligand therapy of metastatic castration-resistant prostate cancer: safety and efficacy. J Nucl Med. 2016;57:1006-1013.

5. Kratochwil C, Giesel FL, Stefanova M, et al. PSMA-targeted radionuclide therapy of metastatic castration-resistant prostate cancer with Lu-177 labeled PSMA-617. J Nucl Med. 2016;57:1170-1176.

6. Rahbar K, Bode A, Weckesser M, et al. Radioligand therapy with ${ }^{177}$ Lu-PSMA617 as a novel therapeutic option in patients with metastatic castration resistant prostate cancer. Clin Nucl Med. 2016;41:522-528.

7. Rahbar K, Schmidt M, Heinzel A, et al. Response and tolerability of a single dose of ${ }^{177} \mathrm{Lu}$-PSMA-617 in patients with metastatic castration-resistant prostate cancer: a multicenter retrospective analysis. J Nucl Med. 2016;57:1334-1338.

8. Ahmadzadehfar H, Eppard E, Kurpig S, et al. Therapeutic response and side effects of repeated radioligand therapy with ${ }^{177}$ Lu-PSMA-DKFZ-617 of castrateresistant metastatic prostate cancer. Oncotarget. 2016;7:12477-12488.

9. Scher HI, Fizazi K, Saad F, et al. Increased survival with enzalutamide in prostate cancer after chemotherapy. N Engl J Med. 2012;367:1187-1197.

10. Ryan CJ, Smith MR, Fizazi K, et al. Abiraterone acetate plus prednisone versus placebo plus prednisone in chemotherapy-naive men with metastatic castrationresistant prostate cancer (COU-AA-302): final overall survival analysis of a randomised, double-blind, placebo-controlled phase 3 study. Lancet Oncol. 2015;16:152-160.

11. Parker C, Nilsson S, Heinrich D, et al. Alpha emitter radium-223 and survival in metastatic prostate cancer. $N$ Engl J Med. 2013;369:213-223.

12. de Bono JS, Oudard S, Ozguroglu M, et al. Prednisone plus cabazitaxel or mitoxantrone for metastatic castration-resistant prostate cancer progressing after docetaxel treatment: a randomised open-label trial. Lancet. 2010;376:1147-1154. 
13. Kantoff PW, Higano CS, Small EJ, Whitmore JB, Frohlich MW, Schellhammer PF. Re: interdisciplinary critique of sipuleucel-T as immunotherapy in castration-resistant prostate cancer. J Natl Cancer Inst. 2012;104:1107-1109, author reply 1109-1112.

14. Petrylak DP, Tangen CM, Hussain MH, et al. Docetaxel and estramustine compared with mitoxantrone and prednisone for advanced refractory prostate cancer. N Engl J Med. 2004;351:1513-1520.

15. Kratochwil C, Stefanova M, Mavriopoulou E, et al. SUV of $\left[{ }^{68} \mathrm{Ga}\right] D O T A T O C-$ PET/CT predicts response probability of PRRT in neuroendocrine tumors. Mol Imaging Biol. 2015;17:313-318.

16. Haug AR, Auernhammer CJ, Wangler B, et al. ${ }^{68}$ Ga-DOTATATE PET/CT for the early prediction of response to somatostatin receptor-mediated radionuclide therapy in patients with well-differentiated neuroendocrine tumors. J Nucl Med. 2010;51:1349-1356.

17. Kaemmerer D, Trager T, Hoffmeister M, et al. Inverse expression of somatostatin and CXCR4 chemokine receptors in gastroenteropancreatic neuroendocrine neoplasms of different malignancy. Oncotarget. 2015;6:27566-27579.

18. Santoni M, Scarpelli M, Mazzucchelli R, et al. Targeting prostate-specific membrane antigen for personalized therapies in prostate cancer: morphologic and molecular backgrounds and future promises. J Biol Regul Homeost Agents. 2014;28:555-563.

19. Bensalah K, Leray E, Fergelot P, et al. Prognostic value of thrombocytosis in renal cell carcinoma. J Urol. 2006;175:859-863.

20. Gakis G, Todenhofer T, Stenzl A. The prognostic value of hematological and systemic inflammatory disorders in invasive bladder cancer. Curr Opin Urol. 2011;21:428-433.

21. Yu M, Liu L, Zhang BL, et al. Pretreatment thrombocytosis as a prognostic factor in women with gynecologic malignancies: a meta-analysis. Asian Pac J Cancer Prev. 2012;13:6077-6081.
22. Gücer F, Tamussino K, Keil F, Balkanli-Kaplan P, Yuce MA. Thrombocytosis in gynecologic malignancies. Anticancer Res. 2004;24:2053-2059.

23. Stone RL, Nick AM, McNeish IA, et al. Paraneoplastic thrombocytosis in ovarian cancer. N Engl J Med. 2012;366:610-618.

24. Voutsadakis IA. Thrombocytosis as a prognostic marker in gastrointestinal cancers. World J Gastrointest Oncol. 2014;6:34-40.

25. Taucher S, Salat A, Gnant M, et al. Impact of pretreatment thrombocytosis on survival in primary breast cancer. Thromb Haemost. 2003;89:1098-1106.

26. Stravodimou A, Voutsadakis IA. Pretreatment thrombocytosis as a prognostic factor in metastatic breast cancer. Int J Breast Cancer. 2013;2013:289563.

27. Pearlstein E, Ambrogio C, Karpatkin S. Effect of antiplatelet antibody on the development of pulmonary metastases following injection of CT26 colon adenocarcinoma, Lewis lung carcinoma, and B16 amelanotic melanoma tumor cells into mice. Cancer Res. 1984;44:3884-3887.

28. Karpatkin S, Pearlstein E. Role of platelets in tumor cell metastases. Ann Intern Med. 1981;95:636-641.

29. Honn KV, Tang DG, Crissman JD. Platelets and cancer metastasis: a causal relationship? Cancer Metastasis Rev. 1992;11:325-351.

30. Zabrenetzky VS, Kohn EC, Roberts DD. Suramin inhibits laminin- and thrombospondin-mediated melanoma cell adhesion and migration and binding of these adhesive proteins to sulfatide. Cancer Res. 1990;50:5937-5942.

31. Francis JL, Biggerstaff J, Amirkhosravi A. Hemostasis and malignancy. Semin Thromb Hemost. 1998;24:93-109.

32. Hejna M, Raderer M, Zielinski CC. Inhibition of metastases by anticoagulants. J Natl Cancer Inst. 1999;91:22-36. 